\title{
Projeção da dinâmica da floresta natural de Terra- firme, região de Manaus-AM, com o uso da cadeia de transição probabilística de Markov
}

Liliane Martins TEIXEIRA, Jeffrey Q. CHAMBERS, Anabel Rodrigues e SILVA, Adriano José Nogueira LIMA, Vilany Matilla Colares CARNEIRO, Joaquim dos SANTOS, Niro HIGUCHI ${ }^{1}$

\section{RESUMO}

Os fatores que envolvem os processos da dinâmica da floresta influenciam a sua biodiversidade e, portanto, a qualidade da floresta. A definição de estratégias que envolve a proteção e o uso adequado da floresta manejada e a recuperação de áreas já degradadas tornam-se possível com o estudo da estrutura e dinâmica da floresta primária por meio de informações como a mortalidade, o recrutamento e a permanência das árvores no sistema florestal. Este trabalho teve como objetivo avaliar a dinâmica de uma floresta não perturbada e fazer projeções da dinâmica florestal usando a matriz de transição probabilística (Cadeia de Markov). As taxas de recrutamento, mortalidade e incremento foram determinadas a partir de inventários florestais realizados em dois transectos, nos sentidos Norte-Sul e Leste-Oeste ( 20 x $2500 \mathrm{~m}$ cada, totalizando $10 \mathrm{ha}$ ), localizados no $\mathrm{km} 50 \mathrm{da}$ BR 174, na estrada vicinal ZF-2, Manaus/AM, nos anos de 2000 e 2004. A floresta acumulou 8,34 t.ha ${ }^{-1}$.ano ${ }^{-1}$ de biomassa fresca acima do solo. De acordo com projeção para 2008, o número total de árvores diminuirá em 2,67\% (de 5987 indivíduos (2004) para 5827 (2008)) e a mortalidade será 15\% maior (de 264 (2004) para 311 (2008)). O teste Qui-quadrado mostrou que não há diferença significativa ( $1 \%$ de probabilidade) entre as informaçôes coletadas e projetadas. Esses resultados permitem concluir que a Cadeia de Markov é um eficiente instrumento para projetar a dinâmica da floresta natural, contribuindo para o planejamento em curto prazo das atividades que utilizam os recursos florestais.

PALAVRAS-CHAVE

Biomassa, Recrutamento, Mortalidade, Incremento.

\section{Dynamics of the Terra-firme primary forest in Manaus-AM region using the Markov probabilistic transition matrix}

\begin{abstract}
To combine protection and utilization of forest resources in the tropics, the understanding of forest dynamics is essential. It is also important in the definition of strategies for rehabilitation of degraded areas. In Forestry, forest dynamics could be translated as the understanding of recruitment, mortality and biomass increment rates over time. For this study, these rates were estimated based on measurements carried out in 2000 and 2004 over two transects measuring 20 by $2500 \mathrm{~m}$ (5 hectares) each, in Manaus region. This paper deals with forest dynamics of a pristine forest based on the probabilistic transition matrix (the first-order Markov Chain) approach. The main objective is to report 4-year (2000 to 2004) changes in the forest structure. Diameter distribution and tree mortality will be projected ahead to $2008(t+2)$, based upon a 4-year period of observations completed in $2004(\mathrm{t}+1)$ and its immediate past in $2000(\mathrm{t})$. In terms of fresh aboveground biomass, this site accumulated $8.34 \mathrm{t} \mathrm{ha}^{-1}$. ano $^{-1}$. The $\chi^{2}$ test has shown no statistical difference $(\mathrm{p}=0.01)$ between observed diameter frequency and the expected projected by Markov Chain. This result indicates that the Markov Chain approach is a reliable tool to project the forest dynamics on a short-term basis. In 2008, the total number of individuals will have a decrease of $2.7 \%$, and the mortality rate will $15 \%$ higher than in 2004 .
\end{abstract}

KEYWORDS

Biomass, Mortality, Recruitment, Increment 


\section{INTRODUÇÃO}

Estudar a dinâmica da floresta tropical úmida amazônica, manejada ou não, é um grande desafio para os florestais. Os modelos clássicos de produção florestal foram desenvolvidos para florestas temperadas e têm como principais variáveis, o índice de sítio e idade da árvore ou do povoamento (Sullivan \& Clutter, 1972; Ferguson \& Leech, 1978; Alder, 1980; Smith, 1983; Clutter et al., 1983). Essas duas variáveis são limitantes para o desenvolvimento de modelos de produção para as florestas da Amazônia porque são praticamente indisponíveis para o setor florestal, num curto prazo. Apesar de inúmeras tentativas, por meio da dendrocronologia ou da datação com ${ }^{14} \mathrm{C}$, a determinação das idades das inúmeras espécies que ocorrem numa determinada área, continua sendo um grande obstáculo para a ciência florestal.

Sem a idade da árvore ou do povoamento ou com muita dificuldade para obtê-la, a alternativa é prognosticar a dinâmica da floresta com o uso de parcelas permanentes. Na Amazônia, entretanto, as parcelas instaladas e devidamente monitoradas são poucas, mal distribuídas e recentes (as mais antigas estão na Flona de Tapajós, desde 1978). Considerando que as idades de árvores com DAP $>50 \mathrm{~cm}$, na região de Manaus, podem variar de 200 a 1400 anos, segundo Chambers et al. (1998), 20-30 anos de observaçóes podem parecer insuficientes para descrever, com confiança, a dinâmica de uma floresta da Amazônia.

Apesar de todas as dificuldades, aproximadamente um milhão de hectares de floresta amazônica são manejados anualmente, para produção madeireira sob algum tipo de manejo em regime de rendimento sustentável. A alternativa pode estar no uso de modelos de curto prazo que dependem exclusivamente da situação imediatamente anterior a atual, tendo como objetivo a projeção apenas para uma situação imediatamente posterior. Dentre os vários modelos disponíveis, o que melhor se ajusta às características das florestas da Amazônia, é a cadeia de Markov.

\section{CADEIA DE MARKOV}

A cadeia de Markov de primeira ordem é um processo estocástico, no qual as probabilidades de transição durante o intervalo de tempo $(\mathbf{t}$ e $\mathbf{t}+\mathbf{1})$ dependem apenas do estado do indivíduo no tempo $\mathbf{t}$ ou no conhecimento do passado imediato no tempo $\mathbf{t}+\mathbf{1}$ e não em qualquer outro estado prévio (Horn, 1975; Chiang, 1980 e Bruner \& Moser, 1973). Shugart (1984) enfatiza que a natureza "invariável em tempo" de cada uma das probabilidades de transição é uma importante característica da cadeia de Markov, tendo muita afinidade com o comportamento dos ecossistemas florestais. De acordo com Bierzychudek (1982), um modelo de matriz de transição é um modelo classificado em tamanho ou uma forma da matriz de Leslie. A única exigência deste modelo é divisibilidade da população em grupo de estados e que existam probabilidades de movimento de um estado para outro, com o passar do tempo (Enright \& Ogden, 1979).

Shugart \& West (1981) apontam que a importância do entendimento dos ecossistemas florestais não é baseada nas idades, mas sim nas mudanças conhecidas no presente. Os modelos determinísticos consistindo de uma simples função matemática (linear, polinomial ou exponencial) não demonstraram ainda que sejam comprovadamente adequados, quando séries de tempo são envolvidas (Morrison, 1976).

Segundo Enright \& Ogden (1979), nas florestas tropicais, o atributo tamanho pode ser mais importante do que a idade. Uma razão para isso é que o tamanho pode ser mais ecologicamente informativo do que a idade, quando esta é difícil de ser obtida com precisão. Além disso, segundo ainda os mesmos autores, a divisão de ciclos de vida em estágios de desenvolvimento pode permitir a predição do comportamento futuro mais precisamente do que a divisão em puras classes de idade. Usher (1966) usou o atributo tamanho no lugar da idade para desenvolver um modelo para o manejo de recursos renováveis. Ele afirma que um organismo que está na i-ésima classe no tempo $\mathbf{t}$, pode permanecer na mesma classe, mudar para a classe seguinte (mais de uma classe também) ou morrer, no tempo $\mathbf{t}+\mathbf{1}$.

Os modelos que usam matriz de transição são apropriados para análise de muitos problemas biológicos, principalmente em estudos relacionados com a dinâmica da floresta (Enright \& Ogden, 1979). Esses modelos têm sido usados intensivamente em estudos de dinâmica de populações de plantas ou animais em várias regiōes do mundo. Alguns exemplos são: a demografia do jack-in-the-pulpit em Nova York (Bierzychudek, 1982); dinâmica florestal de uma população de Araucaria numa floresta tropical úmida de Papua Nova Guinea e Nothofagus em floresta montana temperada da Nova Zelândia (Enright \& Ogden, 1979); sucessão de térmitas em Gana (Usher, 1979); sucessão florestal na Nova Jersey (Horn, 1975); aplicação da Cadeia de Markov em estudos de dinâmica florestal em florestas tropicais (Acevedo, 1981) e a aplicação de Markov para predizer o desenvolvimento de um povoamento florestal (Usher, 1966; Bruner \& Moser, 1973; Peden et al., 1973; Buogiorno \& Michie, 1980).

Alder (1980) também descreve a matriz de transição como uma possível ferramenta para análise de dados de crescimento e incremento de povoamentos multianos de florestas tropicais mistas. Na região de Manaus, Higuchi (1987) usou Markov para estudar a dinâmica da floresta das parcelas testemunhas do projeto de manejo florestal (Projeto Bionte) e Rocha (2001) nos transectos do projeto Jacaranda. A maioria dos trabalhos citados anteriormente inclui revisōes razoáveis da teoria do método de Markov. 
O entendimento do comportamento das taxas de crescimento, recrutamento e mortalidade, em condiçôes naturais ou sob manejo, são importantes para definir quanto tempo uma espécie, ou um grupo de espécies demora em atingir uma determinada dimensão. Essa relação pode definir o ciclo do corte de uma floresta natural, para prescriçôes adequadas de tratamentos silviculturais e indicar se a floresta está respondendo ou não aos tratos silviculturais bem como, indicar se a floresta funciona como sumidouro ou fonte de dióxido de carbono. Este trabalho tem como objetivo avaliar a dinâmica de uma floresta de terra-firme não explorada na região de Manaus, AM, com o uso da cadeira probabilística de transição.

\section{MATERIAL E MÉTODOS}

O estudo foi desenvolvido na Estação Experimental de Silvicultura Tropical do Instituto Nacional de Pesquisas da Amazônia (EEST/INPA), distante aproximadamente $90 \mathrm{~km}$ de Manaus - AM, localizada entre os $\mathrm{km} 21$ e 24 da margem esquerda da estrada vicinal ZF-2 do Distrito Agropecuário da Superintendência da Zona Franca de Manaus (SUFRAMA). As coordenadas geográficas da área estão entre 02³7' e $02^{\circ} 38^{\prime}$ de latitude sul e $60^{\circ} 09^{\prime}$ e $60^{\circ} 11^{\prime}$ 'de longitude oeste, aproximadamente (Radam-Brasil, 1978).

De acordo com a classificação de Köppen, o clima é do tipo "Amw", caracterizado como tropical chuvoso, apresentando temperatura elevada, com variação térmica diurna maior do que a variação térmica anual, com precipitação elevada e predominantemente forte. A temperatura média anual é de $26,7^{\circ} \mathrm{C}$, apresentando valores médios para as máximas e mínimas de $31,2{ }^{\circ} \mathrm{C}$ e $23,5^{\circ} \mathrm{C}$, respectivamente. As chuvas apresentam um total anual da ordem de $2.101 \mathrm{~mm}$, distribuídos em duas épocas distintas durante o ano, uma chuvosa que ocorre entre novembro e maio e a outra que se estende até outubro. A umidade relativa apresenta uma média anual de $84 \%$, variando de 77-88\% (Falesi, 1971).

O solo do tipo Latossolo Amarelo Distrófico e Álico de textura argilosa predomina na região (Radam-Brasil, 1978 \& Chauvel 1982). O relevo é levemente ondulado e a maioria das ondulações é formada por pequenos platôs, que variam de 500 a $1000 \mathrm{~m}$ de altura. A diferença de nível entre as calhas dos igarapés e a superfície dos platôs varia de 70 a $80 \mathrm{~m}$ (Santos, 1996). Ferraz et al. (1998) observaram que a topografia dos transectos apresenta-se disposta em seqüências de platôs, encostas e baixios. Os solos dos platôs apresentaram textura argilosa; nas encostas, variaram de argilo-arenosos, próximos aos platôs à areno-argilosos, próximos aos baixios e, os solos nas áreas de baixio, apresentaram textura arenosa. Os solos nas áreas dos transectos podem ser classificados em três tipos: Latossolo Amarelo nos platôs; Podzólicos Vermelho-Amarelo nas encostas e Arenossolos hidromórficos nos baixios.
A vegetação da área é uma amostra representativa de uma floresta tropical úmida densa de terra-firme amazônica. Radam-Brasil (1978) denominou a área de sub-região dos baixos platôs da Amazônia e, com base na geomorfologia, em levantamentos florísticos e em inventários florestais, classificou a mesma em macro-ambiente de relevo tabular, no qual a cobertura florestal densa, raramente com estrato superior uniforme, é freqüentemente alterada por manchas de floresta aberta, onde os estratos arbustivo e herbáceo são compostos por regeneração natural das espécies arbóreas, palmeiras e plantas não-vasculares. A área apresenta vegetação diversificada com 737 espécies, pertencentes a 238 gêneros de 59 famílias. Dentre as famílias mais ricas em espécies estão Sapotaceae (69 espécies) e Chrysobalanaceae e Lauraceae (44 spp.). As espécies que mais se destacaram foram Oenocarpus bataua Mart., Eschweilera wachenheimii (Benoist) Sandwith e Eperua glabiflora (Ducke) R.S. Cowan (Carneiro, 2004).

Os dados foram coletados em duas toposseqüências (transectos) dispostas no sentido Norte-Sul (Figura 1) e Leste-Oeste (Figura 2) do "Projeto Jacaranda", desenvolvido pela CPST-INPA. Cada transecto possui cinco hectares $(20$ x $2500 \mathrm{~m}$ ), totalizando dez hectares de floresta natural. O acompanhamento da dinâmica da floresta foi iniciado com a realização do inventário de todos os indivíduos arbóreos, onde se realizou a identificação por nome vulgar e medição do diâmetro à altura do peito (DAP) para todos os indivíduos com $\mathrm{DAP} \geq 10 \mathrm{~cm}$.

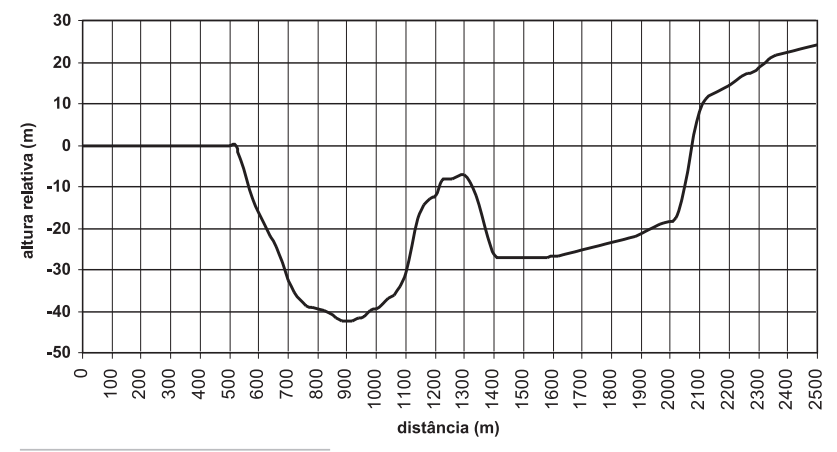

Figura 1 - Perfil Vertical da Toposseqüência do Transecto 1 (Norte - Sul)

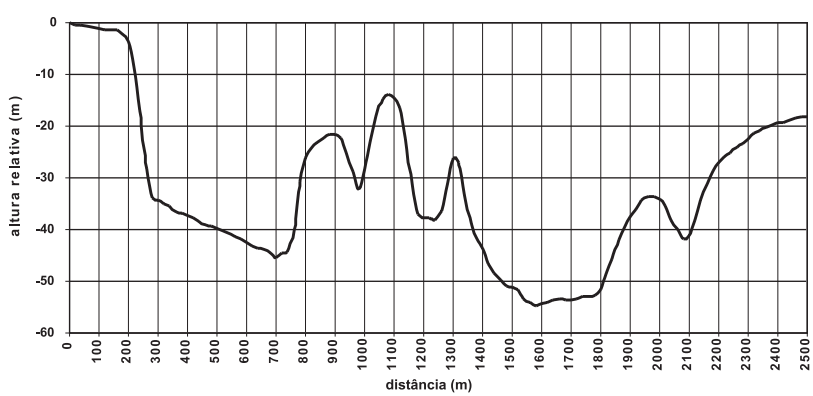

Figura 2 - Perfil Vertical da Toposseqüência do Transecto 2 (Leste - Oeste) 
Para este estudo duas coletas foram necessárias: a primeira foi realizada em 2000, onde se mediu o DAP de cada indivíduo, nos dois transectos. Em 2004, ocorreu a segunda medição de todos os indivíduos já registrados (crescimento em diâmetro), além da inclusão de novos indivíduos que em 2004 passaram a possuir $\mathrm{DAP} \geq 10 \mathrm{~cm}$, entrando para a primeira classe de DAP (Recrutamento) e do registro e identificação das árvores que morreram no período entre 2000 e 2004 (Mortalidade).

A hipótese teste neste trabalho é de que a floresta tropical úmida não explorada, na região de Manaus, pode ser avaliada, com previsōes futuras, com o uso da cadeia de transição probabilística de Markov. A distribuição diamétrica da floresta foi utilizada como variável principal, sendo aplicado o teste Qui-quadrado, para avaliar a hipótese.

O crescimento da floresta foi analisado por meio dos seguintes parâmetros:

a) Área Basal, medida em 2000 e 2004, para cada transecto, pela equação:

$$
\mathbf{G}=\left(\pi \cdot \mathrm{DAP}^{2}\right) / \mathbf{4}
$$

b) Volume Comercial com Casca, estimado pela equação desenvolvida por Higuchi et al. (1997): $0,27]$

Ln $V=-7,335+2,121$ Ln DAP $\left[R^{2}=0,95\right.$ e $S_{y x}=$

c) Biomassa Arbórea Fresca Acima do Nível do Solo, estimada pelas equações ajustadas por Higuchi et al. (1998):

Ln $P=-1,754+2,665$ Ln DAP $\left[R^{2}=0,92\right.$ e $\left.S_{y x}=43\right]$ $(5 \mathrm{~cm} \leq \mathrm{DAP}<20 \mathrm{~cm})$

$\operatorname{Ln} P=-\mathbf{0 , 1 5 1}+\mathbf{2 , 1 7} \operatorname{Ln} \mathrm{DAP}\left[\mathrm{R}^{2}=0,90\right.$ e $\left.\mathrm{S}_{\mathrm{yx}}=2035\right]$ (DAP $\geq 20 \mathrm{~cm}$ ).

d) Mortalidade, obtida pela razão entre o número de indivíduos mortos no período observado, pela abundância de indivíduos arbóreos referente ao ano de 2000:

$\mathrm{TM}=\mathrm{TM} /(\mathrm{NT}-\mathrm{TR})$

e) Recrutamento: foram consideradas como recrutas todas as árvores que na segunda medição (2004) passaram a apresentar $\mathrm{DAP} \geq 10 \mathrm{~cm}$. A taxa de recrutamento foi obtida pela razão entre o número de indivíduos arbóreos ingressantes no período observado e a abundância referente ao número de indivíduos de 2004:

$\mathrm{TR}=\mathrm{TR} /(\mathrm{NT}-\mathrm{TM})$

$\mathrm{G}=$ área basal em $\mathrm{m}^{2} \cdot \mathrm{ha}^{-1}$

$\mathrm{DAP}=$ diâmetro à altura do peito em $\mathrm{cm}$.

Ln $=$ logaritmo natural

$\mathrm{P}=$ peso da biomassa fresca acima do nível do solo, em $\mathrm{kg}$.
TM = Número de indivíduos mortos durante o período observado.

$\mathrm{TR}=$ Número de indivíduos recrutados durante o período observado.

$\mathrm{NT}=$ Número total de indivíduos no período observado.

$\mathrm{VOL}=$ volume em $\mathrm{m}^{3}$

Leste-Oeste $=$ LO

Norte-Sul $=$ NS

\section{RESULTADOS E DISCUSSÃO}

\section{ESTOQUES DE VOLUME, BIOMASSA ARBÓREA E ÁREA BASAL NOS TRANSECTOS LOE NS}

$\mathrm{Na}$ Tabela 1 são apresentados os valores de área basal e volume nos dois transectos (LO e NS) nos anos de 2000 e 2004. A área basal apresentou um valor médio de 51,18 $\mathrm{m}^{2} \cdot \mathrm{ha}^{-1} \pm 3,67 \mathrm{~m}^{2}$.ha $\mathrm{a}^{-1}$ (IC 95\%) e volume médio de 647,94 $\mathrm{m}^{3} \cdot \mathrm{ha}^{-1} \pm 47,08 \mathrm{~m}^{3} \cdot \mathrm{ha}^{-1}$ (IC $95 \%$ ) no ano 2000. E para o ano de 2004, os valores foram de $54,74 \mathrm{~m}^{2}$.ha- $\pm 0,99 \mathrm{~m}^{2}$. ha ${ }^{-1}$ (IC 95\%) para área basal e de $692,77 \mathrm{~m}^{3} \cdot \mathrm{ha}^{-1} \pm 12,17 \mathrm{~m}^{3} \cdot \mathrm{ha}^{-1}$ (IC $95 \%)$ para volume. Os dois transectos tiveram incremento em termos de área basal e volume de $0,44 \mathrm{~m}^{2} \cdot$ ha $^{-1} \cdot$ ano $^{-1} \mathrm{e} \mathrm{5,60}$ $\mathrm{m}^{3} \cdot \mathrm{ha}^{-1} \cdot$ ano $^{-1}$, respectivamente.

Table 1 - Aboveground biomass balance in 10 ha of the primary Amazonian forest (two transects), in Manaus region, during a period of 4 years (2000 to 2004).

\begin{tabular}{|c|c|c|c|c|c|c|}
\hline & 2000 & 2004 & & 2000 & 2004 & \\
\hline Transectos & $\begin{array}{l}A B \\
\left(m^{2} h a^{-1}\right)\end{array}$ & $\begin{array}{l}A B \\
\left(m^{2} h a^{-1}\right)\end{array}$ & $\begin{array}{l}\text { Acúmulo } \\
\left(\mathrm{m}^{2} \mathrm{ha}^{-1} \mathrm{ano}^{-1}\right)\end{array}$ & $\begin{array}{l}\text { VOL } \\
\left(m^{3} h a^{-1}\right)\end{array}$ & $\begin{array}{l}\text { VOL } \\
\left(m^{3} h^{-1}\right)\end{array}$ & $\begin{array}{l}\text { Acúmulo } \\
\left(\mathrm{m}^{3} \mathrm{ha}-\right. \\
\left.{ }^{1} \mathrm{ano}^{-1}\right)\end{array}$ \\
\hline LO & 23,72 & 26,87 & 0,78 & 299,95 & 340,17 & 10,05 \\
\hline NS & 27,46 & 27,87 & 0,11 & 347,99 & 352,59 & 1,15 \\
\hline Total & 51,18 & 54,74 & 0,89 & 647,94 & 692,77 & 11,21 \\
\hline Média & 25,59 & 27,37 & 0,44 & 323,97 & 346,38 & 5,60 \\
\hline
\end{tabular}

Na Tabela 2 são apresentados os estoques e as diferenças de estoques de biomassa vegetal arbórea acima do nível do solo nos dois transectos no período de 2000 a 2004. A estimativa total de biomassa (LO e NS) apresentou um saldo positivo, ou seja, houve acúmulo de biomassa durante o período estudado de 66,71 t.ha ${ }^{-1}$, correspondendo em média a 8,34 t.ha-1.ano ${ }^{-1}$, indicando que a floresta retirou carbono e fixou nas árvores, convertendo-o em tecidos lenhosos, folhas etc. Observando esses valores em média os dois transectos tiveram em $2000 \mathrm{e}$ $2004,485,85 \mathrm{t} \pm 71,82 \mathrm{t}$ (IC 95\%) e 519,21 t $\pm 17,30 \mathrm{t}$ (IC $95 \%)$, respectivamente. 
Tabela 2. Estoque de biomassa fresca acima do nível do solo e diferença de estoques no período de 2000 a 2004, em t ha-1 ano-1.

\begin{tabular}{lllll}
\hline \multicolumn{5}{c}{ Crescimento em Biomassa } \\
\hline Transectos & 2000 & 2004 & Acúmulo & t ha $^{-1}$ ano $^{-1}$ \\
\hline LO & 449,21 & 510,39 & 61,18 & 15,29 \\
NS & 522,50 & 528,03 & 5,54 & 1,38 \\
Total & 971,71 & 1038,42 & 66,71 & 16,68 \\
Média & 485,85 & 519,21 & 33,36 & 8,34 \\
\hline
\end{tabular}

\section{TAXAS DE MORTALIDADE E RECRUTAMENTO}

As causas mais comuns da morte de árvores em florestas tropicais são: idade, estado fitossanitário, estresse hídrico e vento. Em períodos de secas prolongadas (ex. em anos que ocorre o fenômeno El Niño), a mortalidade tende aumentar, porém a maioria das árvores permanece em pé e se decompõem com o passar do tempo. Em períodos de fortes chuvas, as árvores mais frágeis são derrubadas por ação do vento, ocasionando em conseqüência a queda de outras árvores mesmo saudáveis (Rocha et al., 2003).

No período observado (2000-2004), a taxa de recrutamento foi de 3,29\% \pm 0,36\% (IC $95 \%$ ) e a taxa de mortalidade de $2,26 \% \pm 0,22 \%$ (IC 95\%) para os dois transectos. As taxas de recrutamento ( $1,46 \%$ no N-S e $1,83 \%$ no L-O) foram superiores às taxas de mortalidade $(1,02 \%$ e $1,24 \%$ para N-S e L-O, respectivamente). A média anual da taxa de recrutamento foi de $1,65 \%$. Esse valor foi superior às taxas obtidas por Higuchi et al., (1997) e Rocha (2001) (1,1\% e $0,90 \%$, respectivamente), na mesma área de estudo, e a taxas obtidas em outros sítios, como na Costa Rica, 1,8 \% (Lieberman \& Lieberman, 1987), na Malásia, 1,4\% (Manokaran \& Kochummen, 1987) e na Flona Tapajós, 1,4 \% (Carvalho, 1992), porém, foi inferior ao encontrado por Vasconcelos (2003) de 2,3\% no estado do Acre.

Considerando os dois transectos juntos, a taxa de mortalidade média anual foi de 1,13\%, sendo superior às taxas obtidas por Higuchi et al., (1997) e Rocha (2001) (1,03\% e $0,86 \%$, respectivamente) na mesma área de estudo. Porém foi inferior quando comparado a Lieberman \& Lieberman (1987) com 1,8 \% na Costa Rica, Peralta et al., (1987) com $1,8 \%$ a 2,25 \% na Venezuela, a Carey et al., (1994) com 0,5 a 3,3 \% na Malásia, a Carvalho (1992) com 1,3\% na Flona Tapajós e a Vasconcelos (2003), com 2,4 \% no Acre. Durante o período observado, 264 árvores morreram. (média de $132 \pm$ 21,56 árvores por transecto). Em termos gerais, a mortalidade foi superior em $17 \%$ quando comparada com a projeção para 2004. A projeção para mortalidade no período 2000-2004 corresponde, aproximadamente, a $83 \%$ do levantamento realizado em 2004.

\section{PROJEÇÃO DA DISTRIBUIÇÃO DIAMÉTRICA USANDO A CADEIA DE MARKOV}

Os diâmetros em cada ocasiāo foram agrupados em classes com intervalos de $5 \mathrm{~cm}$, onde a primeira classe foi de $10 \mathrm{~cm}$ $\leq \mathrm{DAP}<15 \mathrm{~cm}$ e a última classe diamétrica representada por árvores com DAP $\geq 75 \mathrm{~cm}$.

As matrizes de transição foram montadas com base na distribuição diamétrica das ocasiōes (2000 e 2004). A transição de um estado para outro é obtido quando uma árvore que foi medida em 2000 passa uma ou mais classes seguintes ou morrer, ou ainda permanecer na mesma classe de diâmetro ou estado.

A matriz de transição foi estruturada considerando 17 estados, dos quais o primeiro estado trata-se da classe $\mathrm{R}$ (Recrutamento); do $2^{\circ}$ ao $14^{\circ}$ estados correspondem às 14 classes diamétricas, a partir do DAP $\geq 10 \mathrm{~cm}$; e o $15^{\circ}$ estado (ultima classe diamétrica) que corresponde aos indivíduos com $\mathrm{DAP} \geq 75 \mathrm{~cm}$, podendo variar de $75 \mathrm{~cm}$ até o maior diâmetro medido, sendo, por isso, considerada uma classe aberta. $\mathrm{O} 16^{\circ}$ estado refere-se ao número de árvores, pertencentes à classe de $\mathrm{DAP} \geq 75 \mathrm{~cm}$, que na segunda ocasião (2004) apresentaram crescimento em diâmetro maior que o intervalo de $5 \mathrm{~cm}$, suficiente para a mudança de uma próxima classe, isto é, se a próxima classe existisse. Por exemplo, um indivíduo que em 2000 apresentou DAP de $99 \mathrm{~cm}$ e em 2004 teve o DAP medido em $101 \mathrm{~cm}$, considerando o intervalo de $5 \mathrm{~cm}$, este indivíduo passaria para a próxima classe. E por último, o $17^{\circ}$ estado ou classe M (mortalidade), composto pelas árvores que morreram durante o intervalo de tempo, ou seja, pelas árvores que estiveram presentes na primeira ocasião (2000) e ausentes na segunda ocasião (2004).

A Tabela 3 apresenta a transição de um estado para outro, ocorrida durante o período 2000 - 2004 usando as observações de ambos transectos. Verificou-se que o estado $\mathrm{R}$ obteve 396 árvores, ou seja, 396 indivíduos ingressaram no banco de dados, pois passaram a possuir o DAP $\geq 10 \mathrm{~cm}$. O estado M apresentou 264 indivíduos, ou seja, 264 indivíduos saíram do sistema por causas naturais (fatores ambientais que causaram a sua morte ou senescência). Em geral, as árvores têm maior probabilidade de permanecer nas mesmas classes diamétrica, principalmente considerando um período curto (quatro anos) e a taxa de crescimento anual observada. Por exemplo, a probabilidade associada à permanência de árvores da classe $10<15 \mathrm{~cm}$ no período de 2000-2004 foi de 86,2\%. A mortalidade no período observado na mesma classe foi 4,2\% para o mesmo período. Para essa classe, observa-se que não há um padrão definido, ou seja, as árvores com diâmetros menores têm a mesma probabilidade de morrer que as árvores de diâmetros maiores. De acordo com Rocha (2001), esse comportamento estocástico é típico da floresta tropical úmida, dificultando os trabalhos de modelagem da 
Tabela 3. Transição da freqüência árvores por classe diamétrica (cm), durante o período de 2000 a 2004 em 10ha de floresta natural.

\begin{tabular}{|c|c|c|c|c|c|c|c|c|c|c|c|c|c|c|c|c|c|}
\hline$C D$ & R $10<15$ & $15<20$ & $20<25$ & $25<30$ & $30<35$ & $35<40$ & $40<45$ & $45<50$ & $50<55$ & $55<60$ & $60<65$ & $65<70$ & $70<75$ & $\geq 75$ & Próx. & M & 2000 \\
\hline $\mathrm{R}$ & 385 & 7 & 4 & & & & & & & & & & & & & & 396 \\
\hline $10<15$ & 1869 & 205 & 2 & & & & & & & & & & & & & 91 & 2167 \\
\hline $15<20$ & & 1126 & 144 & 1 & & & & & & & & & & & & 48 & 1319 \\
\hline $20<25$ & & & 711 & 104 & 4 & & 1 & & & & & & & & & 33 & 853 \\
\hline $25<30$ & & & & 419 & 59 & & & & & & & & & & & 24 & 502 \\
\hline $30<35$ & & & & & 276 & 59 & & & & & & & & & & 26 & 361 \\
\hline $35<40$ & & & & & & 195 & 23 & & & & & & & & & 10 & 228 \\
\hline $40<45$ & & & & & & & 119 & 27 & 1 & & & & & & & 8 & 155 \\
\hline $45<50$ & & & & & & & & 72 & 14 & & & & & & & 7 & 93 \\
\hline $50<55$ & & & & & & & & & 36 & 7 & & & & & & 3 & 46 \\
\hline $55<60$ & & & & & & & & & & 28 & 6 & 1 & & & & 6 & 41 \\
\hline $60<65$ & & & & & & & & & & & 19 & 8 & & & & 1 & 28 \\
\hline $65<70$ & & & & & & & & & & & & 12 & 4 & & & 1 & 17 \\
\hline $70<75$ & & & & & & & & & & & & & 15 & 2 & & 1 & 18 \\
\hline$\geq 75$ & & & & & & & & & & & & & & 15 & 7 & 5 & 27 \\
\hline \multicolumn{18}{|l|}{ Próx. } \\
\hline \multicolumn{18}{|l|}{ M } \\
\hline 2004 & 2254 & 1338 & 861 & 524 & 339 & 254 & 143 & 99 & 51 & 35 & 25 & 21 & 19 & 17 & 7 & 264 & 6251 \\
\hline
\end{tabular}

$\mathbf{C D}=$ Classe diamétrica; $\mathbf{R}=$ Recrutamento; $\mathbf{M}=$ Mortalidade; Próx. = Próxima

dinâmica da floresta, principalmente quando se usam modelos determinísticos.

\section{PROJEÇÃO PARA 0 ANO DE 2008}

O resultado final da Cadeia de Markov (Tabela 4) é a projeção da distribuição do número de árvores dos diferentes estados (recrutamento, mortalidade e as classes diamétricas), para um período seguinte de mesmo intervalo de tempo. A projeção para o período seguinte (2004 - 2008) mostrou que a probabilidade dos indivíduos da $1^{\mathrm{a}}$ classe permanecer com $\mathrm{DAP} \leq 15 \mathrm{~cm}$ é de $74,39 \%$. Porém, $16,24 \%$ passarão para a $2^{a}$ classe, $1,19 \%$ passarão para a $3^{\text {a }}$ classe e $0,02 \%$ passarão para a 4 a classe.

A projeção para um período seguinte imediato (2008), com base na transição probabilística do ano de 2004 e a ocasião imediatamente anterior (2000), indica que a taxa de mortalidade será de 250 árvores, bem próxima à observada em 2004 (264 árvores). Para 2008, o número total de árvores será de 5854, sem incluir a taxa de recrutamento, mas se repetida essa taxa (396 recrutas em 2004), o número total de árvores em 2008 será de 6250, 5,6\% maior que o número observado em 2004 (5897).

Para avaliar a qualidade das projeçôes feitas para o ano de 2008 e confirmar o uso da Cadeia de Markov como ferramenta de avaliação para a projeção da dinâmica da floresta tropical e do comportamento futuro deste povoamento florestal, foram comparadas as frequiências projetadas e observadas nos transectos pelo teste Qui-quadrado $\left(\chi^{2}\right)$ ao nível de $1 \%$ e
Tabela 4. Freqüência observada (2000 e 2004) e projetada (2008) de árvores vivas e mortas, por classe de diâmetro e valores de Qui-quadrado $\left(\chi_{\text {cal }}^{2}\right)$. Sendo $\chi^{2}(1 \%)=24,69 ; \chi^{2}(5 \%)=22,36$.

\begin{tabular}{|c|c|c|c|c|c|c|}
\hline \multirow{2}{*}{$\begin{array}{l}\text { Classe } \\
\text { Diamétrica }\end{array}$} & \multicolumn{3}{|c|}{ Freqüência } & \multirow{2}{*}{$\chi_{\text {cal }}^{2}$} & \multicolumn{2}{|c|}{ Mortalidade } \\
\hline & 2000 & 2004 & 2008 & & 2004 & 2008 \\
\hline $10<15$ & 2167 & 2254 & 2243 & 0,05 & 91 & 86 \\
\hline $15<20$ & 1319 & 1338 & 1316 & 0,37 & 48 & 47 \\
\hline $20<25$ & 853 & 861 & 837 & 0,69 & 33 & 33 \\
\hline $25<30$ & 502 & 524 & 519 & 0,05 & 24 & 24 \\
\hline $30<35$ & 361 & 339 & 302 & 4,45 & 26 & 22 \\
\hline $35<40$ & 228 & 254 & 263 & 0,30 & 10 & 10 \\
\hline $40<45$ & 155 & 143 & 128 & 1,71 & 8 & 8 \\
\hline $45<50$ & 93 & 99 & 95 & 0,15 & 7 & 6 \\
\hline $50<55$ & 46 & 51 & 52 & 0,04 & 3 & 3 \\
\hline $55<60$ & 41 & 35 & 27 & 2,18 & 6 & 4 \\
\hline $60<65$ & 28 & 25 & 21 & 0,79 & 1 & 1 \\
\hline $65<70$ & 17 & 21 & 22 & 0,04 & 1 & 1 \\
\hline $70<75$ & 18 & 19 & 20 & 0,02 & 1 & 1 \\
\hline$>=75$ & 27 & 17 & 9 & 7,11 & 5 & 3 \\
\hline Próxima & & 7 & & & & \\
\hline Total & 5855 & 5987 & 5854 * & $\begin{array}{c}17,94 \\
\star *\end{array}$ & 264 & 250 \\
\hline
\end{tabular}

* Sem incluir a taxa de recrutamento. ${ }^{* *}$ Não significativo a 1 \% e 5 \%. 
5\% de significância. De acordo com a Tabela 4, o teste Quiquadrado $\left(\chi^{2}\right)$ mostra que não existe diferença significativa entre as freqüências observadas e projetadas na distribuição do número de árvores em cada classe diamétrica para o ano de $2008\left(\chi_{\text {cal }}^{2}=17,94\right)$. Este resultado confirma a hipótese apresentada neste estudo, indicando que em florestas que apresentam as mesmas características similares às da área amostrada, a Cadeia de Markov pode ser utilizada como instrumento para projetar a dinâmica de povoamentos de florestas naturais, pois contribui para o planejamento em curto prazo de atividades que envolvam a utilização de recursos florestais.

\section{CONCLUSÕES}

O crescimento médio em área basal e volume foram de $0,44 \mathrm{~m}^{2} \cdot \mathrm{ha}^{-1} \cdot$ ano $^{-1}$ e de $5,60 \mathrm{~m}^{3} \cdot$ ha $^{-1} \cdot$ ano $^{-1}$, respectivamente, para uma área de dez hectare de floresta natural de terra-firme no período de 2000-2004. A floresta acumulou em biomassa 8,34 thah $^{-1} \cdot$ ano $^{-1}$, indicando que em condiçôes naturais, a floresta seqüestra carbono, fixando-o para a manutenção, produção e crescimento de novos tecidos. Os valores médios para as taxas de mortalidade e recrutamento foram de 1,13\% e $1,65 \%$, respectivamente.

O teste Qui-quadrado não mostrou diferença significativa entre as freqüências observadas e projetadas para a distribuição do número de árvores em cada classe diamétrica, indicando a Cadeia de Markov como instrumento para a projeção de frequêencia da distribuição diamétrica, do recrutamento e da mortalidade de florestas similares às da região de Manaus, reproduzindo o processo dinâmico das árvores por meio da variável classe diamétrica. A projeção da dinâmica da floresta pode contribuir para o manejo florestal em florestas tropicais na determinação do ciclo de corte ou ainda mostrar as tendências que a floresta apresentará num futuro próximo, para que o técnico ou empreendedor florestal saiba o momento e o volume ideais para manejar a floresta tropical.

\section{BIBLIOGRAFIA CITADA}

Acevedo, M.F. 1981. On Horn's Markovian Model of Forest Dynamics with Particular Reference to Tropical Forests. Theoretical Population Biology, 19: 230-250.

Alder, D. 1980. Forest Volume Estimation and Yield Prediction. V.2 - Yield Prediction. FAO Forestry Paper 22/2. 194pp.

Bierzychudek, P. 1982. The Demography of Jack-in-the-pulpit, a Forest Perennial that Changes Sex. Ecol. Monographs, 52(4):333351.

Bruner, H.D.; Moser Jr, J.W. 1973. A Markov Chain Approach to the Prediction of Diameter Distributions in Uneven-aged Forest Stands. Can.J.For.Res, 3:409-417.

Buogiorno, J.; Michie, B.C. 1980. A Matrix Model of Unever-aged Forest Management. Forest Science, 26:609-625.
Carey, E.V.; Brown, S.; Gillespie, A.J.R.; Lugo, A.E. 1994. Tree Mortality in Mature Lowland Tropical Moist and Tropical Lower Montane Moist Forest of Venezuela. Biotropica, 26(3): 255-264.

Carneiro, V.M.C. 2004. Composição florística e análise estrutural da floresta primária de terra-firme na bacia do rio Cuieiras, Manaus$A M$. Dissertação de Mestrado, Instituto Nacional de Pesquisas da Amazônia/ Universidade Federal do Amazonas, Manaus, Amazonas. 67pp.

Carvalho, J.O.P. 1992. Struture and dynamics of a logger over Brazilian Amazonian rainforest. Doctor of Philosophy's Thesis. University of Oxford. Oxford. 146pp.

Chambers, J.Q.; Higuchi, N; Schimel, J.P. 1998. Ancient Trees in Amazonia. Nature, 391:135-136.

Chauvel, A. 1982. Os latossolos amarelos, álicos, argilosos, dentro dos ecossistemas das bacias experimentais do INPA e da região vizinha. Acta Amazonica, 12(3): 47-60.

Chiang, C.L. 1980. An Introduction to Stochastic Processes and their Applications. Robert E. Krieger Publ. Co., Huntington, New York. 380pp.

Clutter, J.L.; Fortson, J.C.; Pienaar, L.V.; Brister, G.H.; Bailey, R.L. 1983. Timber Management: A Quantitative Approach. John Wiley and Sons, Inc. New York. 333pp.

Enright, N.; Ogden, J. 1979. Applications of Transition Matrix Models in Forest Dynamics: Araucaria in Papua New Guinea and Nothofagus in New Zealand. Australian. J. of Ecology, 4:3-23.

Falesi, I.C. 1971. Solos do Distrito Agropecuário da Suframa. Bol. Instituto de Pesquisa e Experimentação Agropecuária da Amazônia Ocidental. Série: Solos. Manaus, Amazonas.1(1). 99pp.

Ferguson, I.S.; Leech, J.W. 1978. Generalized Least Squares Estimation of Yield Functions. Forest Science, 24: 27-42.

Ferraz, J.; Ohta, S.; Salles, P.C. 1998. Distribuição dos Solos ao Longo de Dois Transectos em Floresta Primária ao Norte de Manaus (AM). In: Higuchi, N; Campos, M.A.A.; Sampaio, P.T.B.; Santos, J. (Eds). Pesquisas Florestais para Conservação da Floresta e Reabilitação de Areas Degradadas da Amazônia.111-143.

Higuchi, N. 1987. Short-term growth of an undisturbed tropical moist forest in the Brasilian Amazon. Doctor of Philosophy's Thesis, Michigan State University, Michigan. 129p.

Higuchi, N. 1994. Utilização e Manejo dos Recursos Madeireiros das Florestas Tropicais Úmidas. Acta Amazonica, 24(3-4): 275 288.

Higuchi, N.; Santos, J.; Ribeiro, R.J.; Freitas, J.V. de; Vieira, G.; Cöic, A.; Minette, L.J. 1997. Crescimento e Incremento de uma Floresta Amazônica de Terra-Firme Manejada Experimentalmente. In: Relatório Final do Projeto Bionte. Biomassa e Nutrientes Florestais - MCT/INPA. Manaus. 89-131.

Higuchi, N.; Santos, J.; Ribeiro, R.J.; Minette, L.J.; Biot, Y. 1998. Biomassa da parte aérea da vegetação da Floresta Tropical Úmida de Terra-Firme da Amazônia Brasileira. Acta Amazonica, 28 (2): 153-166.

Horn, H.S. 1975. Markovian Properties of Forest Succession. In: M. Cody e J. Diamond, Eds. Ecology and Evolution of Communities, Harvard University Press. Cambridge, Mass. 196-211. 
Lieberman, D.; Lieberman, M. 1987. Forest tree growth and dynamics at La Selva, Costa Rica $(1969$ - 1982). J. of Trop. Eco, 3:347-358.

Manokaran, N.; Kochummen, K.M. 1987. Recruitment, grow and mortality of trees in a lowland dipyterocarp forest in Peninsular Malaysia. J. of Trop. Eco, 3:315-330.

Morrison, D.F. 1976. Multivariate Statistical Methods. McGrawHill Inc.. 415pp.

Peralta, R.; Hartshorn, G.S.; Lieberman, D.; Lieberman, M. 1987. Reseña de estudios a largo plazo sobre composición florística y dinámica del bosque tropical en La Selva, Costa Rica. In: Clark, D.A.; Dirzo, R.; Fetcher, N. (Eds). Ecologia y ecofisiología de plantas en los bosques mesoamericanos. Revista de Biologia Tropical, 35 (1). 23-40.

Peden, L.M., Williams, J.S.; Frayer, W.E. 1973. A Markov Model for Stand Projection. Forest Science, 19: 303-314.

Radam-Brasil. 1978. Programa de Integração Nacional. Levantamento de Recursos Naturais. V. 18 (Manaus) RADAM (projeto) DNPM, Ministério das Minas e Energia. Brasil. 626pp.

Rocha, R.M. 2001. Taxas de Recrutamento e Mortalidade da Floresta de Terra Firme da Bacia do Rio Cuieiras na Região de ManausAM. Dissertação de Mestrado. Instituto Nacional de Pesquisas da Amazônia/Universidade Federal do Amazonas, Manaus, Amazonas. 49pp.

Rocha, R.M.; Higuchi, N.; Santos, J.; Nakamura, S.; Silva, R.P.; Pinto, A.C.M.; Tribuzy, E.S. 2003. Taxas de recrutamento e mortalidade e mudanças de estoques de fitomassa da floresta primária na região de Manaus-Am. In: Higuchi, N; Santos, J. dos; Sampaio, P. B. T.; Marenco, R. A.; Ferraz, J.; Sales, P.C.; Saito, M.; Matsumoto, S. (Eds). Pesquisas Florestais na Amazônia Central. Projeto Jacaranda Fase II. 43-54.
Santos, J. 1996. Análise de modelos de regressão para estimar a fitomassa da floresta tropical úmida de terra-firme da Amazônia brasileira. Tese de Doutorado, Universidade Federal de Viçosa. Viçosa, Minas Gerais. 120pp.

Shugart, H.H.; West, D.C. 1981. Long-term Dynamics of Forest Ecosystems. Am. Scientist., 69:647-652.

Shugart, H.H. 1984. A Theory of Forest Dynamics: The Ecological Forest Succession Models. Springer-Verlag Inc. New York. 278p.

Smith, V.G. 1983. Compatible Basal Area Growth and Yield Models Consistent with Forest Growth Theory. Forest Science, 29:279-288.

Sullivan, A.D.; Clutter, J.L. 1972. A Simultaneous Growth and Yield Model for Loblolly Pine. Forest Science, 18:76-86.

Usher, M.B. 1966. A Matrix Approach to the Management of Renewable Resources, with Special Reference to Selection Forests. J. of Applied Ecology. 3(2): 355-367.

Usher, M.B. 1979. Markovian Approaches to Ecological Succession. J. of Animal Ecology, 48: 413-426.

Vasconcelos, S.S. 2003. Dinâmica de uma floresta explorada seletivamente no Projeto de Colonização Pedro Peixoto na Amazônia Ocidental. Dissertação de Mestrado. Instituto Nacional de Pesquisas da Amazônia/Universidade Federal do Amazonas, Manaus, Amazonas. 71pp.

Recebido em 09/08/2006

Aceito em 08/02/2007 